\title{
Kinematic study of biped locomotion
} systems

\author{
F.M. Pereira da Silva
}

Modern University, Dept. of Control and Automation

Rua Augusto Rosa 24, 4000 Porto, Portugal

Tel: 3512 2026938, Fax: 3512 2026939,E.mail: fpsilva@fe.up.pt

\section{J.A. Tenreiro Machado}

Faculty of Engineering of the University of Porto, Dept. of Electrical and Computer Engineering

Rua dos Bragas, 4099 Porto, Portugal

Tel: 3512 317105, Fax: 3512 319280,E.mail:jtm@fe.up.pt

\begin{abstract}
This paper presents the kinematic study of robotic biped locomotion systems. The main purpose is to determine the kinematic characteristics and the system performance during walking. For that objective, the prescribed motion of the biped is completely characterised in terms of five locomotion variables: step length, hip height, maximum hip ripple, maximum foot clearance and link lengths. In this work, we propose four methods to quantitatively measure the performance of the walking robot: energy analysis, perturbation analysis, lowpass frequency response and locomobility measure. These performance measures are discussed and compared in determining the robustness and effectiveness of the resulting locomotion.
\end{abstract}

\section{Keywords}

Biped locomotion, locomotion variables, kinematics, motion planning, performance measure 


\section{INTRODUCTION}

In recent years a growing field of research in biped locomotion has resulted in a variety of prototypes whose characteristics, in limited sense, resemble the biological systems (Golden, 1990; Kajita, 1994). This new generation of innovative robotic systems, for real-world applications, is bringing up fascinating perspectives in research and development work. The control of legged vehicles is a difficult and multidisciplinary problem and, even today, is regarded as the most crucial aspect of locomotion (Hemami, 1983). Vukobratovic et al. (1990) have proposed models and mechanisms to explain biped locomotion. On the other hand, Raibert and his colleagues built various biped walking machines. Extensive considerations of the major issues with dynamic balance have been presented by Raibert (1986) and Todd (1985). In another perspective, Galhano et al. (1992) demonstrated the appeal of implementing biological-inspired schemes in the analysis of mechanical systems. The theories and algorithms guiding the biological research suggested specific models to apply in biped robots.

In this line of thought, several researchers are in pursuit of better walking robots and continue to refine their models of locomotion (Kun, 1996; Yamaguchi, 1996). The main objective is to design and control an optimum leg geometry that provides good energy efficiency, the required working volume and simplicity in the structure. The movement of the robotic biped will be produced by joint actuators and will be moderated by environmental aspects. Therefore, the resultant motion depends on those factors influencing the structural and functional characteristics of the robot (kinematics problem) and those related to physical phenomena, such as gravity, friction and reaction forces (dynamics problem).

This paper concentrates in the kinematic study of a planar biped model with six degrees of freedom and rotational joints. The main purposes are threefold:

- To gain insight into the phenomenon of biped walking.

- To characterise the biped motion in terms of a set of locomotion variables.

- To establish the correlation among these locomotion variables and the system performance.

A wide variety of gait patterns are developed by prescribing the cartesian trajectories of the hip and the lower extremities of the leg. For the analysis of the resulting motion, various kinematic performance measures are proposed and discussed. These kinematic indices can be regarded as quantitative measures of the biped's ability in transporting the section of the body from an initial position to a desired position through the action of the lower limbs. The performance measures and the associated graphical results are used for evaluating the different locomotion patterns.

The remainder of the paper is organised as follows. A short description of the biped model is given in section 2 . In section 3 we describe the method used to plan the kinematic trajectories of the biped robot. In section 4 various kinematic performance measures are proposed and discussed mathematically. Given this background, several simulation examples are presented in section 5 to illustrate the application of these methods. Finally, in section 6 we outline the main conclusions and the perspectives for future research. 


\section{BIPED MODEL}

Figure 1 shows the planar biped model and the conventions used throughout this paper.

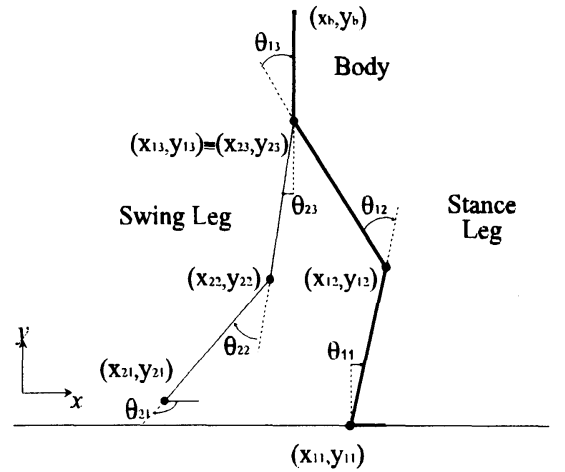

Figure 1 Planar biped model.

The kinematic model consists of seven links in order to approximate locomotion characteristics similar to those of the lower extremities of the human body (i.e., body, thigh, shank and foot). In the present study, we consider a planar biped model with rigid links interconnected through rotational joints. During locomotion, the stance leg (i.e., the limb that is on the ground) has a degree of freedom formed in the contact between the foot and the ground. A step in the gait of a biped consists of two phases:

- Single support phase in which one leg is in contact with the ground and the other leg swings forward.

- Exchange of support in which the legs trade role.

In the single support phase, the stance leg is in contact with the ground and carries the weight of the body, while the swing leg moves forward in preparation for the next step. The impact of the swing leg is assumed to be perfectly inelastic and the friction is sufficient to ensure that no slippage occurs. Additionally, we consider that the support shifts instantaneously from one limb to the other.

\section{FORWARD MOTION PLANNING}

A fundamental problem in robotics is to determine the trajectories that allow the biped robot to walk more skilfully (Shih, 1993). In early work, the determination of the biped trajectories was made largely on the basis of experience and intuition (e.g., recording kinematic data from human locomotion (Silva, 1996)). In this work, the motion planning is accomplished by prescribing the cartesian trajectories of the body and the lower extremities of the leg. Furthermore, the prescribed motion is completely characterised in terms of five locomotion variables. Simultaneously, we determine the relation between the locomotion variables and the trajectories physically admissible. In this context, we introduce the concept of locomotion workspace. 


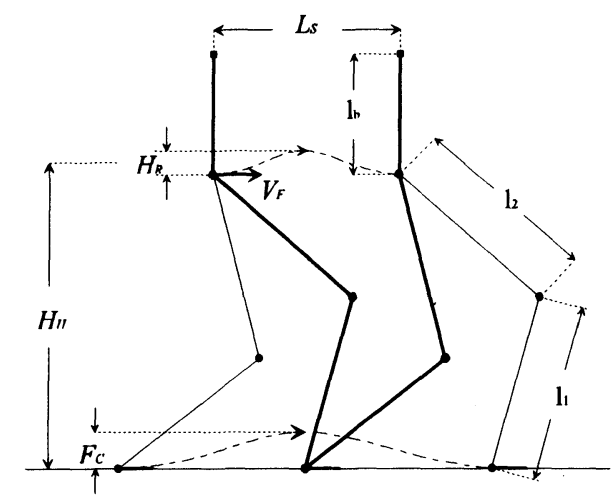

Figure 2 Locomotion variables.

\subsection{Locomotion variables}

The prescribed motion of the biped is completely characterised in terms of the following variables: length of the step $L_{S}$, hip height $H_{H}$, maximum hip ripple $H_{R}$, maximum foot clearance $F_{C}$ and leg link lengths $l_{1}$ and $l_{2}$ (see Figure 2). The hip height is defined as the mean height of the hip along the cycle of walking. The hip ripple is measured as the peak-topeak oscillation magnitude. During the experiments, we examine the role of the link lengths considering that $l_{1}+l_{2}$ assumes a constant value equal to 1 meter.

\subsection{Development of the kinematic trajectories}

The proposed algorithm accepts the hip and feet's trajectories as inputs and, by means of an inverse kinematics algorithm, generates the related joint trajectories. To improve the smoothness of the motion we impose two additional restrictions: the body maintains an erect posture during locomotion and the forward velocity is constant. Therefore, the horizontal trajectory of the hip is implemented using a constant progression velocity $V_{F}$. One trajectory that undergoes smooth motion is the flat trajectory in which the stance leg adjusts itself so that the hip maintains a constant height. Simultaneously, it is of interest to exploit trajectories in which de hip translates with some vertical motion. In order to simplify the problem, we consider that such motions are produced based on sinusoidal functions.

In dynamic walking, at each footfall, the system may suffer impacts and incurs on additional accelerations that influence the forward velocity (Zheng, 1984). For this reason, we must impose a set of conditions (continuity conditions) on the leg velocities so that the feet are placed on the ground while avoiding the impacts. We denote the moment of exchange of support as time $t_{1}$, and by $t_{1}{ }^{-}$and $t_{1}{ }^{+}$the times before and after the impact occurs, respectively. For smooth exchange-of-support, we require the angular velocities, before and after, to be identical, that is:

$\dot{\theta}_{2 i}\left(t_{1}\right)=\dot{\theta}_{1 i}\left(t_{1}^{+}\right) \quad 1 \leq i \leq 3$ 
The kinematic relations have been used and the differential problem solved to obtain the cartesian velocities immediately before and after contact. These equations constrain the biped to zero velocity foot falls. The foregoing derivation determined conditions for smooth exchange-of-support. In accordance, the equation of the tip of the swing leg along the $x$-axis is computed by summing a linear function with a sinusoidal function:

$x_{21}(t)=2 V_{F}\left[t-\frac{1}{2 \pi f} \sin (2 \pi f t)\right]$

where $f$ is the step frequency (number of steps per unit of time). Moreover, the vertical motion, that allows the foot to be lifted off the ground, is implemented using the function:

$y_{21}(t)=\frac{F_{C}}{2}[1-\cos (2 \pi f t)]$

The trajectory generator is responsible for producing a motion that synchronises and coordinates the leg behaviour. In this perspective, we assure that the swing limb arrives at the contact point when the upper body is properly centred with respect to the two lower limbs.

\subsection{Workspace analysis}

One pertinent question involving the motion planning is to determine which trajectories are physically realisable. Upon this point, we define the locomotion workspace of the biped robot as the set of all physically possible hip heights and step lengths, relative to a given reference trajectory (see Figure 3). The kinematic workspace, as defined above, can be investigated by solving the kinematic equations given two kinds of restrictions.

- Sinusoidal restrictions due the type of velocity and acceleration trajectories used.

- Knee-foot restrictions since the legs are constrained by ground contact and the knee joints lock at certain positions (similar to human knee).

In this way, all the results derived ahead are capable of displaying the performance measure for interpretation over the entire stand-up workspace.

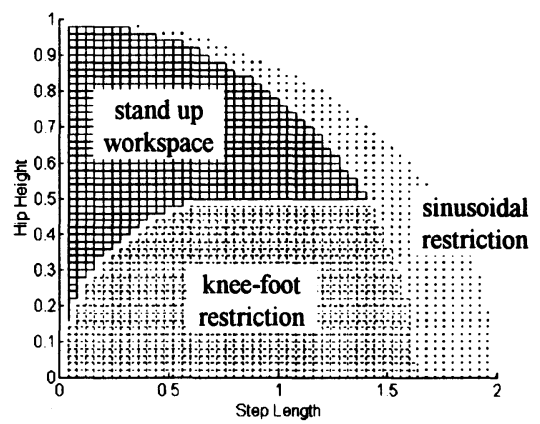

Figure 3 Workspace boundaries of the biped robot with $l_{1}=l_{2}=0.5$. 


\section{PERFORMANCE EVALUATION}

This section covers the implementation of different performance measures used in the evaluation of the biped's kinematic performance. The kinematic analysis is a challenging problem since the biped motion is characterised by gait patterns with complexity of large degrees of freedom (i.e., multidimensional problem). In mathematical terms, we shall provide four global measures of the overall dexterity of the mechanism in some average sense. The aim is to verify whether a correlation between different viewpoints could be found in walking.

\subsection{Energy analysis}

The essence of locomotion is to move smoothly the section of the body from one place to another with some restrictions in terms of execution time. This makes the forward velocity $V_{F}$ an important variable in determining the walking performance. On the other hand, the objective function should be expressed in the coordinate system on which movement control occurs. This appears to favour the use of joint coordinates to calculate the performance measure.

In this line of thought, we intent to determine the energy characteristics of the rotational-tolinear motion system. This motion conversion is concerned with taking the rotational motion from the joint actuators and producing a linear motion at the body. As a means of inferring the energy flow, we suppose that the translational system exhibits a fictional friction acting on the tip of the body. The linear relationship between the force and the velocity is given by,

$$
F(t)=B v_{b}(t)
$$

where $B$ is the viscous-friction coefficient. For purposes of analysis, suppose that the value of the fictitious friction coefficient is constant $B=1 \mathrm{Ns} / \mathrm{m}$. The velocity at the body can be obtained, as function of the joint variables, by differentiating the forward kinematic equations:

$$
\begin{aligned}
& \dot{x}_{b}=\Gamma_{X_{1}}(\theta) \cdot \dot{\theta}_{11}+\Gamma_{X_{2}}(\theta) \cdot \dot{\theta}_{12}+\Gamma_{X_{3}}(\theta) \cdot \dot{\theta}_{13} \\
& \dot{y}_{b}=\Gamma_{Y_{1}}(\theta) \cdot \dot{\theta}_{11}+\Gamma_{Y_{2}}(\theta) \cdot \dot{\theta}_{12}+\Gamma_{Y_{3}}(\theta) \cdot \dot{\theta}_{13}
\end{aligned}
$$

In order to assure an erect posture of the body, the transfer relationships between the joint space and the operational space, along the $x$ and $y$-axis, are expressed by:

$$
\begin{aligned}
& \Gamma_{X_{1}}(\theta)=l_{1} \cos \theta_{11}+l_{2} \cos \left(\theta_{11}+\theta_{12}\right)+l_{b} \\
& \Gamma_{X_{2}}(\theta)=l_{2} \cos \left(\theta_{11}+\theta_{12}\right)+l_{b} \\
& \Gamma_{X_{3}}(\theta)=l_{b}
\end{aligned}
$$

and

$$
\begin{aligned}
& \Gamma_{Y_{1}}(\theta)=-l_{1} \sin \theta_{11}-l_{2} \sin \left(\theta_{11}+\theta_{12}\right)-l_{b} \\
& \Gamma_{r_{2}}(\theta)=-l_{2} \sin \left(\theta_{11}+\theta_{12}\right)-l_{b} \\
& \Gamma_{r_{3}}(\theta)=0
\end{aligned}
$$


For the case of a force acting in the same direction as the velocity of the body, the average power may be defined as the product of the force and the average velocity of the body, that is,

$$
\begin{aligned}
& P_{x}=\frac{1}{T} \int_{0}^{T} \dot{x}_{b}^{2}(t) d t \\
& P_{y}=\frac{1}{T} \int_{0}^{T} \dot{y}_{b}^{2}(t) d t
\end{aligned}
$$

where $\dot{x}_{b}^{2}$ and $\dot{y}_{b}^{2}$ are related with the joint angular velocities by the following equations:

$$
\begin{aligned}
& \dot{x}_{b}^{2}=\sum_{i=1}^{3} \Gamma_{X_{i}}^{2}(\theta) \cdot \dot{\theta}_{1 i}^{2}+\sum_{j=1}^{3} \sum_{k=1, k \neq j}^{3} \Gamma_{X_{j}}(\theta) \cdot \Gamma_{X_{k}}(\theta) \cdot \dot{\theta}_{1 j} \cdot \dot{\theta}_{1 k} \\
& \dot{y}_{b}^{2}=\sum_{i=1}^{3} \Gamma_{Y_{i}}^{2}(\theta) \cdot \dot{\theta}_{1 i}^{2}+\sum_{j=1}^{3} \sum_{k=1, k \neq j}^{3} \Gamma_{Y_{j}}(\theta) \cdot \Gamma_{Y_{k}}(\theta) \cdot \dot{\theta}_{1 j} \cdot \dot{\theta}_{1 k}
\end{aligned}
$$

The biped structure presents a nonlinear relationship between the input joint's rotation and the output's linear displacements. Consequently, some terms in the final equations (13) and (14) are not of simple physical interpretation. The first term in summation represents the energy transferred by each individual joint $\left(P_{x_{i}}\right.$ and $\left.P_{y_{i}}\right)$. The second term corresponds to product velocity terms associated with energy coupling $\left(P_{x_{j k}}\right.$ and $\left.P_{y_{j k}}\right)$. The purpose of the energy analysis method is to determine the input-output characteristics of the biped system and, if possible, to unmask its detailed structure. This involves finding the locomotion variables that move the locomotion system in a desired manner and to minimise the energy expended.

\subsection{Perturbation analysis}

In many practical cases the robotic system is noisy, that is, has internal random disturbing forces. As such, an approach called "perturbation analysis" was implemented to determine how the biped model has enough flexibility to match these distortions. First, the joint trajectories are computed by the inverse kinematics algorithm. Afterwards, the angular acceleration vectors are 'corrupted' by additive noise. For simplicity reasons, it is used a uniform distribution with zero mean added to the acceleration signal. As result, the trajectories of the body suffer some distortion and can only approximate the desired one. By regarding the forward kinematics of the mechanism, we can determine a two-dimensional index based on the statistical average of the well-known mean-square-error and defined by:

$$
\begin{aligned}
& \xi_{x}=\frac{1}{N_{S}} \sum_{i=1}^{N_{S}} \sqrt{\frac{1}{T} \int_{0}^{T}\left[\dot{x}_{b}^{i}(t)-\dot{x}_{b}^{d}(t)\right]^{2} d t} \\
& \xi_{y}=\frac{1}{N_{S}} \sum_{i=1}^{N_{S}} \sqrt{\frac{1}{T} \int_{0}^{T}\left[y_{b}^{i}(t)-y_{b}^{d}(t)\right]^{2} d t}
\end{aligned}
$$


Here, $N_{s}$ is the total number of steps for averaging purposes, $\dot{x}_{b}^{i}$ and $\dot{x}_{b}^{d}$ are the $i$ th sample and the desired forward velocities at the body section, respectively, and $y_{b}^{i}$ and $y_{b}^{d}$ are the $i$ th sample and the desired vertical oscillations at the body. In this method, the stochastic perturbation penalises the system's performance and we shall be concerned with minimising both indices $\xi_{x}$ and $\xi_{y}$.

\subsection{Lowpass frequency response}

In robotics, the electro-mechanical system that regulates the movement (e.g., actuators and drives) is constrained by its bandwidth. An important point to remember is that the bandwidth limits the maximum speed with which a system can respond to an input command. Hence, the practical conditions of motor control should also be considered when evaluating the system's performance.

In this perspective, the joint velocities are expanded in Fourier series and the time domain signals are described by the coefficients of their harmonic terms. Afterwards, these series are truncated to the lower harmonic components by a lowpass filter, which passes a certain portion of the frequency spectrum while neglecting all the higher harmonics. In doing so, the response of the system will lag behind the input command, thereby producing a tracking error. Once again, we can compare the real and the desired trajectories produced at the body using, as our figure of merit, the mean-square-error as expressed in equations (17) e (18) for $N_{s}=1$. This method, based on frequency domain analysis, has also been successfully employed. However, it is a less general method than the perturbation analysis, because it requires the a priori knowledge about the nature of the robotic actuators.

\subsection{Locomobility measure}

The motivation that led to the development of the locomobility index was to apply the concepts of kinematic dexterity to biped walking. This performance measure can be expressed, in mathematical terms, using the geometrical representation of the kinematics through the Jacobian matrix (Park, 1994). In our case, we selected a two-dimensional index of the global directional uniformity of the motion based on the projections of the major axis of the ellipsoid. The global index is obtained by averaging the longest projections of the major axis along the $x$ axis $\left(E_{P_{x}}\right)$ and the $y$-axis $\left(E_{P_{y}}\right)$, over a complete cycle:

$$
\begin{aligned}
& L_{x}=\frac{1}{T} \int_{0}^{T} E_{P_{x}}(t) d t \\
& L_{y}=\frac{1}{T} \int_{0}^{T} E_{P_{y}}(t) d t
\end{aligned}
$$

The more suitable trajectory is that which maximises $L_{x}$ (direction of movement) and minimises $L_{y}$. 


\section{SIMULATION RESULTS}

In this section, we perform a set of experiments to estimate how the biped robot adapts to variations in the locomotion variables. The aim is to determine the main factors that optimise the leg motion and to compare the formulated dexterity measures. To evaluate the system's operating features, the simulations are carried out considering a forward velocity $V_{F}=1$ $\mathrm{m} / \mathrm{sec}$.

\subsection{Step Length and Hip Height}

To illustrate the use of the preceding results, the biped locomotion was simulated. At this phase, a major simplification is introduced by allowing the swing foot stays on the ground for all time without violating any constraint. The individual indices are computed while maintaining a constant hip height during the stride. As shown in Figure 4, the performance function is evaluated with respect to the step length and the hip height (for equal link lengths). In this case, we are plotting the average power in terms of $x$ and $y$ coordinates. For convenience, the surface portion corresponding to hip height values less than $0.5 \mathrm{~m}$ is not represented.

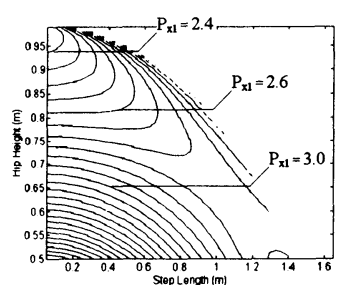

(a)

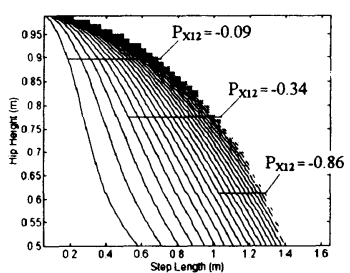

(d)

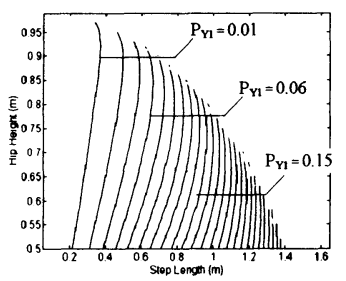

(g)

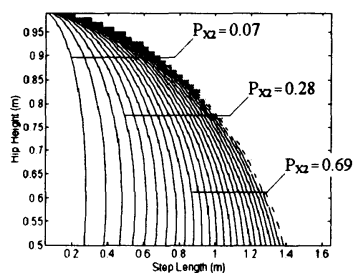

(b)

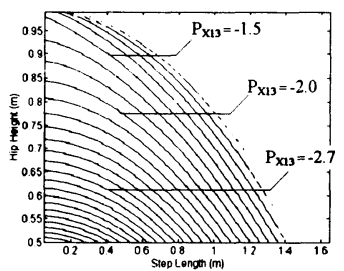

(e)

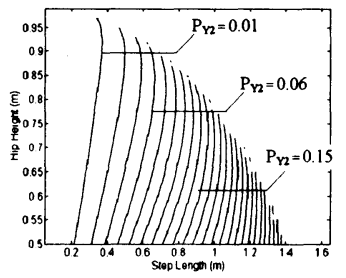

(h)

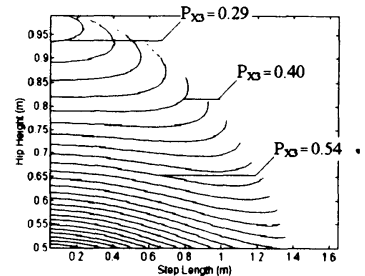

(c)

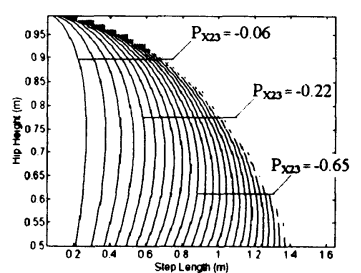

(f)

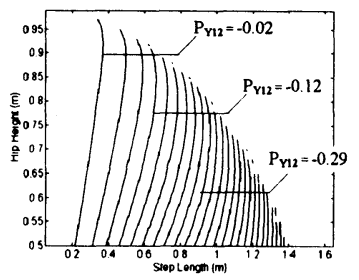

(i)

Figure 4 Contour plot of the average power along a complete cycle of walking. 
The ankle joint is believed to dominate the process of walking, as seen from the energy values involved in every joint (Figure $4(a-c)$ ). The simulation, also, indicates that the knee joint may be a major factor due a significant range of performance variation. As shown in Figures $4(b)$ and $4(h)$, an important degradation occurs as the step length value increases. On the other side, the hip seems to be particularly sensitive to the conditions of hip height (Figure 4(c)). In what concerns the cross coupling terms (Figures $4(d-f)$ and $4(i))$, the average power along a complete cycle resulted in negative values.

Figure $5(a)$ corresponds to the perturbation analysis method. Considering these plots, we conclude that an important degradation occurs as the step length increases. The hip height variable also affects the measure, however, has a smaller influence. In Figure 5(b), we depict the results of applying the locomobility index. The basic idea to retain is that to maximise $L_{x}$ and, additionally, to minimise $L_{y}$, the hip height is expected to round about $95 \%$ of the maximum height.
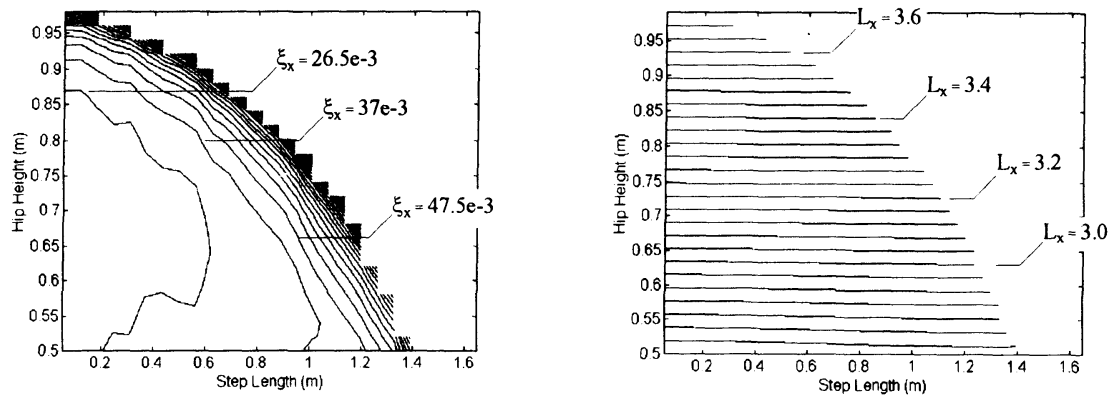

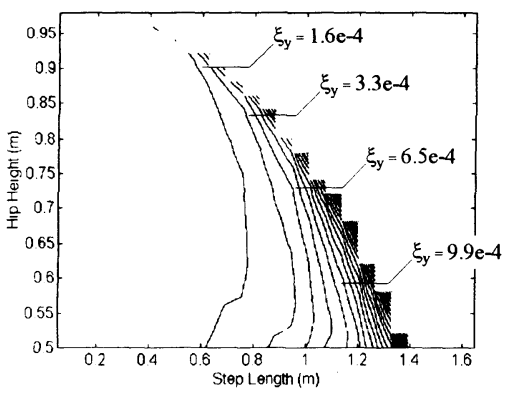

(a)

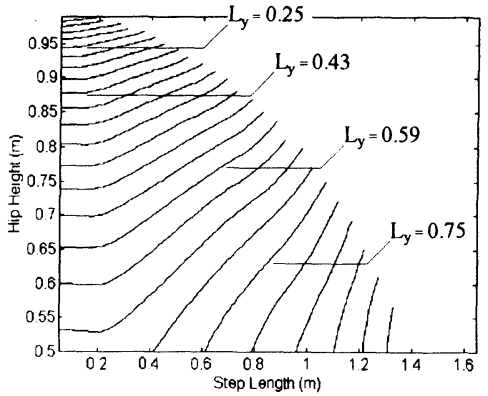

(b)

Figure 5 Contour plot of the performance surface along the $x$ and $y$-axis: (a) perturbation analysis; (b) locomobility index.

Finally, let us examine the "lowpass frequency response" method. The fundamental harmonic $f_{0}$ of the waveforms at the joints is given by,

$f_{0}=\frac{V_{F}}{2 L_{S}}$ 


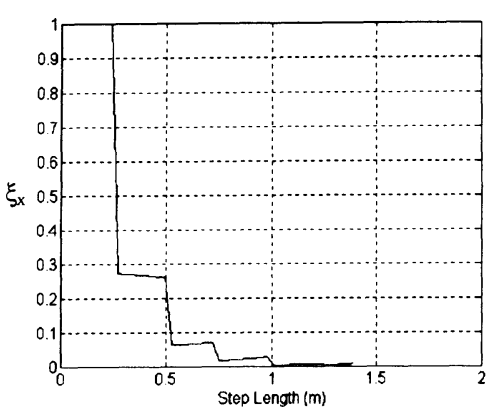

(a)

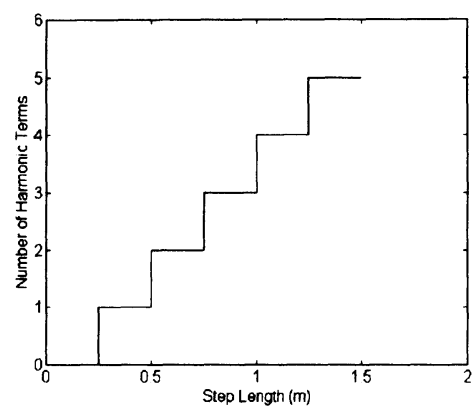

(b)

Figure 6 Lowpass frequency response: $(a)$ mean-square error $\xi_{x}$ versus step length; $(b)$ number of harmonic terms.

If, for example, we assume an ideal lowpass filter whose cutoff frequency is $f_{c}=2 \mathrm{~Hz}$, the performance surface presents a set of discontinuities, as illustrated in Figure $6(a)$. These discontinuity points solely depend on the number of harmonic terms up to $f_{c}$. The number of lower harmonics passing the ideal filter versus the step length is shown in Figure $6(b)$. In contrast to the other methods, the above results suggest that there is an optimum solution for higher step length values, while the performance remains almost unchanged to hip height variations. In order to achieve broader applicability, we must provide information about the robotic actuators by means of a mathematical model (e.g., frequency response).

\subsection{Hip Ripple}

Upon this point, we consider a hip trajectory with a sinusoidal oscillation. In this case, the foot of the swing leg remains at the ground for all cycle. The contour plot in Figure 7 displays the performance values using the perturbation analysis method. From this simulation, we can observe that the optimum hip ripple tends to a zero value and, furthermore, it remains almost unchanged to hip height variations.
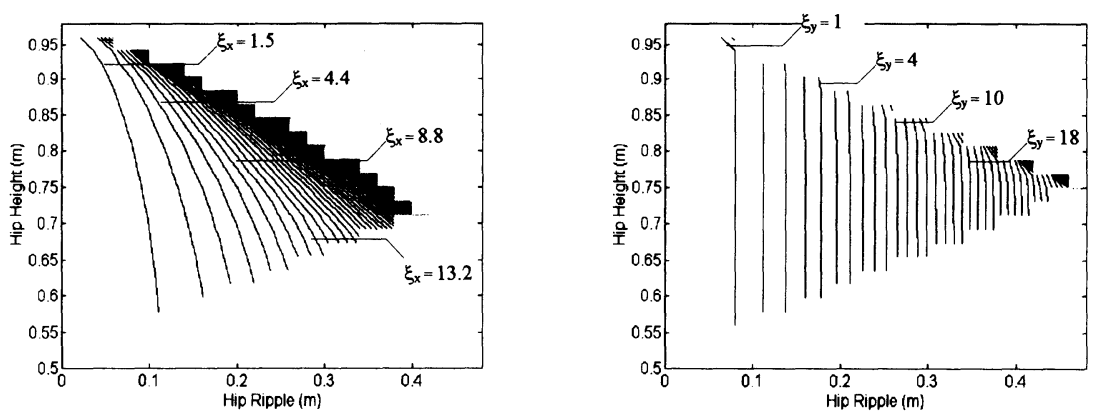

Figure 7 Contour plot: $H_{H}$ versus $H_{R}\left(L_{S}=40 \mathrm{~cm}\right)$. 


\subsection{Foot Clearance}

Another variable that should be tested is the effect of foot clearance. Until now, all the experiences considered that the foot stays on the ground without any restriction. Next, the goal is to analyse the situation in which the foot can be lifted off the ground. In Figure 8 we study the influence of $F_{C}$ for different hip height conditions. The results can be expressed as contours of constant performance measure values in terms of $H_{H}$. We confirmed this invariance property of the foot clearance using the locomobility analysis.
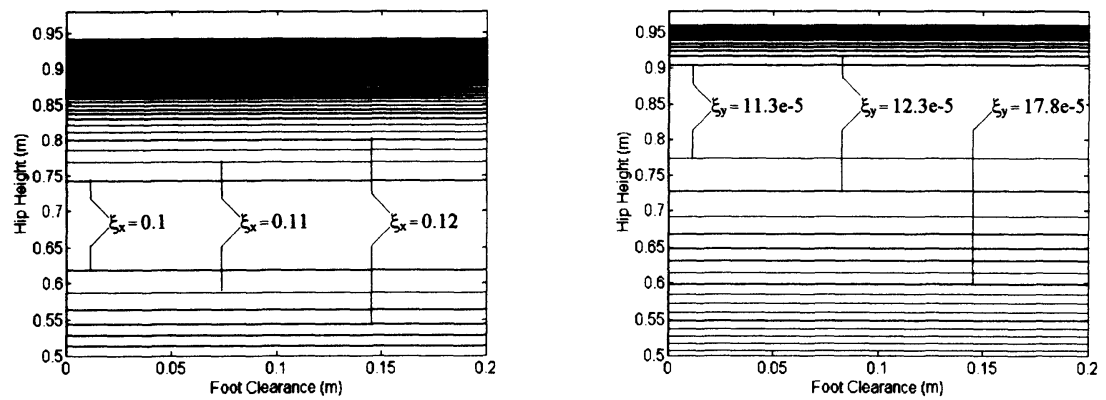

Figure 8 Contour plot: $H_{H}$ versus $F_{C}\left(L_{S}=40 \mathrm{~cm}\right)$.

\subsection{Relative Link Lengths}

We now investigate the role of the link lengths, $l_{1}$ and $l_{2}$, in the system's performance. The choice of the leg lengths is a relevant aspect at the design process and affects the robot's mobility, as well. Figure 9 depicts the curve of $\xi_{x}$ given the link length $l_{1}$, when employing the perturbation method. As can be observed, the optimum solution occurs when the knee-ankle length is higher $\left(l_{1}=0.74 \mathrm{~cm}\right)$ than the hip-knee length $\left(l_{2}=0.26 \mathrm{~cm}\right)$. The simulation also indicates that for $l_{1}$ in the range from 40 to $90 \mathrm{~cm}$ the performance index remains almost constant. The variation found along the $y$-axis is insignificant and is not represented.

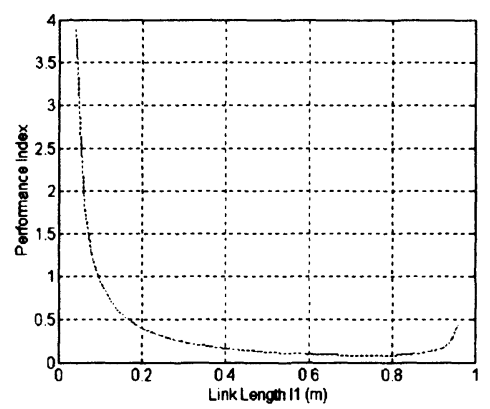

Figure 9 Performance curve versus link length $l_{1}$. 


\section{CONCLUSIONS}

Nowadays, robots are widely used in the manufacture industry. However, the applications of these industrial robots are relatively simple and, for the most part, involving repetitive tasks previously performed by human beings. Bringing robots out of engineered environments and striving for increased autonomy, reliability and dexterity, is both an enormous challenge and a socio-economic necessity in the near future. One key component required to build such intelligent systems is the locomotor system. Accordingly, the biped locomotion aspects of robotics are gaining more and more attention in both research and applications.

In this paper, we have compared various kinematic aspects of biped locomotion. By implementing different motion patterns, we estimated how the robot responds to a variety of locomotion variables such as hip ripple, hip offset, foot clearance and relative link lengths. Various quantitative measures were formulated for analysing the kinematic performance of such systems. Given these goals, the graphical results provide a more concrete illustration of the system's properties. The energy analysis method has the advantage that is very amenable to computer implementation and, furthermore, it is computationally more efficient. However, this approach has the drawback that the resulting equations are not in a completely intuitive form. In general, the perturbation analysis method tends to be more elegant although computationally more exigent. Its random characteristic seems to be particularly tailored for examining the role of the different variables on the locomotion process. On the other hand, the results obtained using the locomobility measure present a correlation with that of the perturbation analysis. The lowpass frequency response produces basically different effects allowing a different perspective of the problem. Moreover, some knowledge of the actuator's characteristics will always be helpful when evaluating the system's performance.

While our focus has been on kinematic dexterity, certain aspects of locomotion are not necessarily captured by the measures proposed. Thus, future work in this area will address the refinement of our models to incorporate dynamics, as well as exploring human-like walking principles. In contrast to rotational actuators used in robotics, the skeletal is completely controlled by linear actuators organised in agonist-antagonist pairs. A complementary analysis could reveal some important properties of both approaches giving some hints to understand the performance differences between robotic and biological motion.

\section{REFERENCES}

Basmajian, J. and Luca, C. (1978) Muscles Alive: Their Functions Revealed by Electromyography. Williams \& Wilkins.

Galhano, A.M., Tenreiro Machado, J.A. and Martins de Carvalho, J.L. (1992) Statistical Analysis of Muscle-Actuated Manipulators. IEEE International Conference on Robotics and Automation, Nice.

Golden, J.A. and Zheng, Y.F. (1990) Gait Synthesis for the SD-2 Biped Robot to Climb Stairs. International Journal of Robotics and Automation, vol. $5, \mathrm{n} .4$.

Hemami, H. and Stokes, B. (1983) A Qualitative Discussion of Mechanisms of Feedback and Feedforward in the Control of Locomotion. IEEE Transactions on Biomedical Engineering, vol. 30, n. 11, 681-688. 
Kajita, S. and Tani, K. (1994) Study of Dynamic Biped Locomotion on Rugged Terrain: Theory and Basic Experiments. Proceedings of '94 International Conference on Advanced Robotics, 741-746.

Kun, A. and Miller, W.T. (1996) Adaptive Dynamic Balance of a Biped Robot using Neural Networks. Proceedings of the IEEE International Conference on Robotics and Automation, 240-245.

Park, F.C. and Brockett, R.W. (1994) Kinematic Dexterity of Robotic Mechanisms. The International Journal of Robotics Research, vol. 13, n. 1, 1-15.

Raibert, M.H. (1986) Legged Robots that Balance. The MIT Press.

Shih, C-L., Gruver W.A. and Lee T-T. (1993) Inverse Kinematics and Inverse Dynamics for Control of a Biped Walking Machine. Journal of Robotic Systems, vol. 10, n. 4, 531-555.

Silva, F.M. and Tenreiro Machado, J.A. (1996) Research Issues in Natural and Artificial Biped Locomotion Systems. Proceedings of 2nd. Portuguese Conference on Automatic Control, 211-216, Controlo'96.

Todd, D.J. (1985) Walking Machines: An Introduction to Legged Robots. Kogan Page.

Vukobratovic, M., Botovac, B., Surla, D. and Stokic D. (1990) Biped Locomotion: Dynamics, Stability, Control and Applications. Springer-Verlag.

Yamaguchi, J. et al. (1996) Development of a Dynamic Biped Walking System for HumanoidDevelopment of a Biped Walking Robot Adapting to the Humans' Living Floor. Proceedings of the IEEE International Conference on Robotics and Automation, 232-239.

Zheng, Y.F. and Hemami, H. (1984) Impact Effects of Biped Contact with the Environment. IEEE Transactions on Systems. Man and Cybernetics, vol. 14, n. 3, 437-443. 